\title{
1 Epigenetic Effects of Assisted Reproductive Technology in Human Offspring
}

3 Wei Chen ${ }^{1,2,3,4, \uparrow}$, Yong Peng ${ }^{1,2,3,4, \uparrow}$, Xinyi Ma ${ }^{1,2,3,4, \uparrow}$, Siming Kong ${ }^{1,2,3,4}$, Shuangyan Tang ${ }^{1}$,Yuan

$4 \mathrm{Wei}^{1}$,Yangyu Zhao ${ }^{1}$, Wenxin Zhang ${ }^{1}$, Yang Wang ${ }^{1,2,3, *}$, Liying Yan $^{1,2,3, *}$, Jie Qiao ${ }^{1,2,3,4,5, *}$

\section{Affiliations}

$7 \quad{ }^{1}$ Center for Reproductive Medicine, Department of Obstetrics and Gynecology Third Hospital, 8 Academy for Advanced Interdisciplinary Studies, Peking University, Beijing 100871, China.

$9 \quad{ }^{2}$ Key Laboratory of Assisted Reproduction, Ministry of Education, Beijing 100191, China.

$10{ }^{3}$ Beijing Key Laboratory of Reproductive Endocrinology and Assisted Reproductive Technology, 11 Beijing 100191, China.

$12{ }^{4}$ Peking-Tsinghua Center for Life Sciences, Peking University, Beijing 100871, China.

$13{ }^{5}$ Beijing Advanced Innovation Center for Genomics, Peking University, Beijing 100871, China.

$14 \uparrow$ These authors contributed equally to this work.

15 *Corresponding author: yangwang@bjmu.edu.cn, yanliyingkind@aliyun.com,

16 jie.qiao@263.net 


\section{Abstract}

The births of more than 8 million infants have been enabled globally through assisted reproductive technologies (ARTs), including conventional in vitro fertilization (IVF) and intracytoplasmic sperm injection (ICSI) with either fresh embryo transfer (ET) or frozen embryo transfer (FET). However, the potential for elevated risks of ART-related disorders persists in adult life, and the underlying epigenetic mechanisms are largely uncharacterized. Here, we recruited 100 nuclear families and profiled the DNA methylomes, genome-wide histone modifications and transcriptomes to clarify the inherent extra risks attributable to specific ART procedures. We discovered that IVF-ET seemed to introduce less disturbance into the infant epigenome than IVFFET or ICSI-ET did. Furthermore, we noted approximately half of the DNA methylomic changes in ART-conceived offspring could be explained by parental background biases. Through removal of the parental effect, we confirmed that ART per se would introduce minor DNA methylation changes locally. More importantly, we found that ART-induced epigenomic alterations were highly enriched in the processes which might contribute to increased incidence of preeclampsia during pregnancy and metabolic syndrome in offspring. Overall, our study provides an epigenetic basis for the potential long-term health risks in ART-conceived offspring that reinforces the need to review all methods of human ART. 


\section{Introduction}

Assisted reproductive technology (ART) has become routine in infertile treatment; indeed, more than eight million ART-conceived infants have been born worldwide ${ }^{1}$. However, conventional in vitro fertilization and fresh embryo transfer (IVF-ET) will introduce extraordinary changes in the environment where oocytes mature and the early embryo develops ${ }^{2}$. Moreover, intracytoplasmic sperm injection (ICSI), which was initially used to address severe male infertility, has replaced IVF as the most commonly used method for ART-mediated fertilization in many countries $^{3}$. This more invasive fertilization procedure introduces additional mechanical damage, bypasses the complicated process of sperm-egg recognition and alters a series of downstream reactions ${ }^{4}$. Embryo cryopreservation enables embryos to be preserved for further transplantation, but both cryogens and freeze-thawing operation may cause damage to embryos ${ }^{5}$. All those unfavorable factors have raised concerns regarding the long-term health of ART-conceived children in recent years ${ }^{6}$. Despite claims to the contrary ${ }^{7-9}$, accumulating evidences have linked ART with potentially increased risks of neurodevelopmental disorders, cardiovascular dysfunction and metabolic abnormity in offspring and preeclampsia during pregnancy ${ }^{10,11}$.

Epigenetic modifications, such as DNA methylation and histone modifications, play key roles in regulating gene expression and are relatively sensitive to environmental factors ${ }^{12}$. Preimplantation embryos undergo dramatic genome-wide epigenetic reprogramming ${ }^{13,14}$ that coincides with the time frame of ART treatments. Thus, ART-associated perturbations may disturb the establishment and maintenance of epigenomic patterns and increase the relevant health risks of ART-conceived children in later life ${ }^{15}$. Notwithstanding, published researches on the association 
57 between ART and DNA methylation in offspring are limited to either specific genes ${ }^{16,17}$ or repeated

58 sequences $^{18}$ and restricted by methods ${ }^{19-23}$. Meanwhile, few studies have parsed the parental

59 inheritance bias where considerable reports have revealed that parental genetic backgrounds,

60 health situations, nutritional conditions and living habits have potential impacts on the epigenomes

61 of neonates ${ }^{24,25}$. In addition, genome-wide changes in histone modifications of ART-conceived

62 infants have not been reported and the effects of each specific ART procedure have not been fully

63 elucidated so far.

Here, we integrated genome-wide maps of DNA methylation, four histone modifications

associated with promoter/enhancer function (H3K4me1, H3K4me3, H3K27ac and H3K27me3),

and gene expression for nuclear families to investigate the specific multilayer effects of various

ART procedures on epigenomes in offspring. We found that various ART treatment would not

dramatically disturb the global epigenome of neonates but subtly induced local and functional

serve as a valuable resource for researchers on the epigenetic influences of ART procedures.

Results

Global epigenomic profiles in ART-conceived neonates

To systematically study the DNA methylomic effects of different types of ARTs on offspring,

77 (UCB) samples and 158 parental peripheral blood (PPB) samples from nuclear families with either 
singletons or twins (Fig. 1a, Supplementary Figure. 1a and Supplementary Table 1). The samples

were classified into five groups based on the mode of conception: spontaneous (CTRL), IVF-ET,

IVF-FET, ICSI-ET and ICSI-FET. There were no significant differences in the clinical features of

(Fig. 1a; Supplementary Figure. 1a and Supplementary Table 1). 
and transcriptomes of neonates in general but that $\mathrm{H} 3 \mathrm{~K} 4 \mathrm{me} 3$ might be exceptionally sensitive to disturbance by specific ART procedures.

Correlation analysis for the DNA methylomes of twin pairs showed an overall high correlation coefficient greater than 0.99 (Fig. 1d); the coefficient for monozygotic twins was significantly higher than that for dizygotic twins in the CTRL group, consistent with previous studies $^{26,27}$. The relatively lower correlation coefficients in all four ART subgroups than in the CTRL group implied that ART processes might subtly affect the epigenomes of neonates (Fig. 1d). Additionally, this phenomenon could also be observed when only same-sex twins were analyzed or in any two non-twin-pair neonates in each group (with exclusion of twin-pair bias) (Supplementary Figure. 3a-c). Moreover, the standard deviations of the DNA methylation levels of all neonates in the different ART groups were slightly but significantly higher than that in the CTRL group (Fig. 1e). Above results indicated that ART itself might increase heterogeneity in neonates. Intergroup correlation analysis revealed that the IVF-ET group was the most similar to the CTRL group with regard to $\mathrm{H} 3 \mathrm{~K} 4 \mathrm{me} 1, \mathrm{H} 3 \mathrm{~K} 4 \mathrm{me} 3, \mathrm{H} 3 \mathrm{~K} 27 \mathrm{ac}$, transcriptomes and DNA methylomes genome-wide or on special elements (Fig. 1f and Supplementary Figure. 3d), indicating that IVF-ET might have less epigenetic influence on neonates than the other three ART processes. These findings suggested that imperceptible disturbances might be introduced into neonates and might vary among different ART subtypes.

\section{Subtle epigenomic changes in ART-conceived offspring}

To elucidate the epigenetic effects of ARTs on neonates, we performed pairwise comparisons 
120

121

122

123

124

125

126

127

128

129

130

131

132

133

134

135

136

137

138

139

140

between groups for DNA methylation, histone modifications, and gene expression. In particular,

to identify differentially methylated regions (DMRs), six comparisons were generated using all

neonates, only singleton neonates, and four groups of neonates from twin cohorts. DMRs in at

least four comparisons were selected for downstream analysis to improve the confidence of the

analysis (Supplementary Figure. 3e, see Methods for more details). The numbers of hyper-/hypo-

DMRs, gain/loss differential histone modifications (differential peaks, DPs), and up-

/downregulated differentially expressed genes (DEGs) among the different comparison groups are

shown in Fig. $1 \mathrm{~g}$ and Supplementary Table 4 (see Supplementary Table 5-10 for more details). The

difference detected in DMRs was mainly less than $15 \%$, which was relatively moderate

(Supplementary Figure. 4a). Meanwhile, only a few DPs were observed in the comparison between

the IVF-ET and CTRL groups for four histone modifications, suggesting that IVF-ET has little

impact on histone modification (Fig. 1g and Supplementary Table 4). The numbers of H3K4me3

DPs detected in the comparison between the ICSI-ET/IVF-FET and CTRL groups (ICSI-ET: 8352

gain, 205 loss; IVF-FET: 5526 gain, 210 loss) were consistent with the observation in unsupervised

clustering (Fig. 1b and Supplementary Figure. 2a, f-g), while the majority of the increased DPs in

the ICSI-ET/IVF-FET groups were in regions with originally weak signals in the CTRL and IVF-

ET groups (Supplementary Figure. 4b). All of the DEGs, DMRs and DPs for each ART group

versus the CTRL group were further validated at the individual level and broadly distributed on

the genome scale (Supplementary Figure. 4c-e; Supplementary Figure. 5). Together, these results

indicated that ART processes potentially caused subtle epigenetic changes distributed widely

throughout the genome. 
Parent-derived and ART-derived DNA methylomic differences in offspring

To evaluate parental influence on the DNA methylomes of progeny, the DNA methylation

\section{Epigenomic alteration of regulatory regions in IVF-ET-conceived offspring}

IVF-ET, the most basic process of ART treatment, is generally recommended as a first-line 
162

163

164

165

166

167

168

169

170

171

172

173

174

175

176

177

178

179

180

181

182

IVF-ET might lead to abnormal phenotypes in ocular, cardiovascular and skeletal system, tooth morphology and metabolism, etc. (Fig. 3b; Supplementary Table 16).

Given the distinct roles of various genomic regions in regulating gene expression, we performed ChromHMM using four types of histone modifications and identify 12 chromatin states to investigate the specific impacts of IVF-ET on the DNA methylation of functional elements (Fig. 3c). The regions covered in our DNA methylation data were mainly the regions related with transcription start site (TSS) (E7 and E8), consistent with the features of highly enriched CpG regions in RRBS. Notably, a large fraction of DMRs were concentrated on Active enhancer (E2), Poised enhancers (E4), Active TSS upstream sites (E6) and Bivalent/Poised TSSs (E8), but almost depleted from Active TSSs (E7) (Fig. 3d). GO analysis for those DMRs in different chromatin states suggested that those hyper-DMRs in Weak enhancer (E1), Bivalent/Poised TSSs (E8) and ZNF Genes \& Repeats (E9) might be associated with the interference on nervous system, while hyper-DMRs in Repressed Polycomb (E10) might account for the influence on cardiovascular system. Meanwhile, hypo-DMRs in Active TSS upstream (E6) and Weak repressed polycomb (E11) might highly correlated with immune system and skeletal system, respectively (Fig. 3e, Supplementary Table 16). In fact, the intersection of the DMR associated genes with the DEGs revealed that the majority of the DMRs were not associated with transcriptional changes in their associated genes (Fig. 3f, Supplementary Table 17). For DEGs overlapped with hypo-DMRs associated genes, MDGA1 was upregulated in the IVF-ET group, while RGS12 was downregulated; in the former, the DMRs occurred in promoters, while in the latter they occurred in introns. The expression of genes associated with hyper-DMRs in introns or distal intergenic regions, such as 
KIAA1671 (introns), DIP2C, CCND1 and CD28, was decreased (Fig. 3g).

Polymorphisms/mutations in MDGA1 and DIP2C are associated with mental disease, and

furthermore, RGS12 dysfunction contributes to tumorigenesis as well as pathological cardiac

hypertrophy, osteoclast genesis and bone destruction. Together, our findings indicated that DNA

methylomic changes caused by IVF-ET might affect multiple aspects related with neonates' health,

but mainly kept away from active TSS regions.

\section{Identification of altered epigenomic profiles associated with ICSI procedures}

To investigate the differences between those two ways of fertilization, we first compared ICSI-

ET with IVF-ET and identified more than 4500 DMRs and 9000 H3K4me3 DPs (Supplementary

Tables 4 and 11; 2365 hyper-DMRs and 2386 hypo-DMRs; 8914 gain DPs and 270 loss DPs).

Although there were no overlapped genomic locations, the changes shared common associated

genes and GO terms (Extended Data Figs. 7a-c), with only a few genes showed consistent changes

in expression with changes in the epigenome (Supplementary Tables 18). Taking the differences

between the ICSI-ET and CTRL groups into account enabled us to determine the abnormal effects

of ICSI per se. Thereafter, the DMRs and H3K4me3 DPs of the ICSI-ET group versus the IVF-ET

group were refined, the former based on k-means clustering of DNA methylation levels among the

ICSI-ET, IVF-ET and CTRL groups and the latter based on integrated comparison between DPs 
225 H3K4me3 modification raise the question of whether ICSI may have a potential impact on health

226 of offspring, with the skeletal systems and immune system as the representatives.

\section{Specific epigenomic changes induced by freeze-thawing procedure}

We then investigated the differences between the IVF-FET and IVF-ET groups and observed

To further explore the specific effects of freeze-thawing procedure per se, we selected six subgroups of DMRs of IVF-FET vs IVF-ET through k-means clustering of scaled DNA 
were highly enriched in the processes of neutrophil-mediated immunity and the regulation of

247 GTPase/Ras (Fig. 5d). The results of HPO analysis further revealed that the selected DMRs might

248 be related to carcinogenesis (Supplementary Figure. 8d; Supplementary Table 22). The majority

249 of selected hyper/hypo-DMRs were inclined to evade from Active TSS (Fig. 5e), and the

250 associated genes of those DMRs in Active enhancer (E2) and Active TSS upstream (E6) were

251 highly enriched in the processes of immune system, metabolism of purine/carbohydrate, muscle

252 and skeletal system (Fig. 5f; Supplementary Table 22). Poised enhancer (E4) was the most

253 overrepresented states for the selected gain H3K4me3 DPs in CTRL/IVF-ET group and became

ZNF Genes \& Repeats (E9) in IVF-FET. Associated genes for the selected gain H3K4me3 DPs in

E2 and E4 were enriched in the processes of certain systems (Fig. 5e-f; Supplementary Table 22).

It was also worth mentioning that the genes $P G P, P L O D 3$, and STAB1 in the IVF-FET group all later in life. 
(Fig. 6a; Supplementary Table 25; C22, n=931; C23, n=776). The associated genes of C22 and

C23 were highly enriched in the regulation of GTPase activity, stress-activated MAPK cascade, 
288 ET would be expected as a mixed effect of ICSI and freeze-thawing operation, indeed, large

289 percentage of those DMRs showed similar changes in ICSI-ET or IVF-FET (C31 C33; C35, C36,

290 C38). However, 13\% hyper-DMRs (C34) and 19\% hypo-DMRs (C37) were uniquely found in

291 ICSI-FET groups, implying complex interactions between ICSI and freeze-thawing

292 (Supplementary Figure. 9d, Supplementary Table 25). The above results together suggested that

293 in addition to causing their own unique effects, as mentioned previously, distinct ART procedures

294 also leaded to certain common effects on the epigenomes of offspring.

\section{Discussion}

Safety concerns regarding ARTs are as old as ARTs themselves ${ }^{30}$. Although a number of

studies suggest that ART may have adverse effects on the long-term health on offspring, the

underlying mechanisms remain to be elucidated. We systematically explored the influences of

fertilization procedures and freeze-thawing operation on descendant genome-wide DNA

ART-irrelevant parental impacts as much as possible. Our study illustrates that the epigenomes of 
offspring were derived from parents, highlighting the necessity of removing parental bias in

assessing the influences of ARTs per se. Moreover, we reported the genome-wide impacts of

ARTs on four types of histone modifications in humans for the first time. The epigenomes of

IVF-ET conceived infants seemed to be closer to those of naturally conceived infants than those

of IVF-FET or ICSI-ET conceived infants and showed almost no disturbance in either type of

histone modification, suggesting that ART-inherent procedures, including controlled ovarian

hyperstimulation $(\mathrm{COH})$, in vitro culture, in vitro fertilization, etc., may not increase the risks of

abnormal histone reprogramming; however, ICSI and freeze-thawing operation may do so. What

surprised us was that $\mathrm{H} 3 \mathrm{~K} 4 \mathrm{me} 3$ was the most profoundly impacted by ICSI and freeze-thawing

compared with the other three types of histone modifications (H3K4me1, H3K27me3 and

$\mathrm{H} 3 \mathrm{~K} 27 \mathrm{ac}$ ), therefore, $\mathrm{H} 3 \mathrm{~K} 4 \mathrm{me} 3 \mathrm{might}$ serve as a sensitive histone modification for assessment

of the influences of ARTs. 
330 follow-up studies are required. Previous studies have revealed elevated rates of preeclampsia in

331 women who have undergone $\mathrm{FET}^{34}$. GTPases, especially Rho kinases, play essential roles in

332 extravillous trophoblast cell (EVT) invasion ${ }^{35}$, and limited EVT invasion following poor

333 remodeling of arteries is widely observed in preeclampsia ${ }^{36}$. More interestingly, our results

334 suggested that freeze-thawing operation might cause dysregulation in the GTPase/Ras signaling

335 pathway in offspring. Thus, the epigenetic abnormality we report may also possibly explain the

336 increased risks of preeclampsia in FET compared with fresh ET pregnancies. Our findings

337 emphasize that it is reasonable to inform patients of potential risks associated with ICSI and

338 embryo cryopreservation and that it would be wise to reconsider overutilization of ICSI or routine

339 freezing of all embryos.

Apart from the influences for given ART operations, we also revealed the common effects

342 changes were enriched in the processes of cardiovascular system and glucolipid metabolism in the

345 it implies that the disturbance on epigenome by ART in offspring may increase the risk of

346 metabolic syndrome ${ }^{38}$. In addition, we identified a considerable number of common DMRs among

347 different ART groups compared with CTRL group. It is noteworthy that the regulation of GTPase 
351 DMRs induced by both ICSI and freeze-thawing procedure were enriched in the processes

352 involving in neuron, consistent with the concern that these two kinds of aggressive ART operation

353 might increase the risk of mental disorders in offspring ${ }^{41}$. As suggested by the reports that

354 improved in vitro culture systems for animals will affect the epigenome less than earlier

355 versions ${ }^{42,43}$, continuous optimization for ART procedures is urgently needed to simulate the in

356 vivo environment and reduce potential epigenetic abnormalities in offspring.

357 Our study was mainly focused on the effects of different fertilization methods and freeze-

358 thawing and only involved newborns after full-term pregnancy, lacking postnatal follow-up data

359 on the enrolled populations. A larger multicenter randomized controlled trial (RCT) along with

360 detection of the epigenetic profiles of offspring in later life would be helpful for elucidation of

361 continued epigenetic change and exploration of the specific epigenetic impacts of other factors in

362 ART treatment, such as the duration of embryo culture or the composition of the culture system.

363 In conclusion, our results provide an epigenetic basis for the increased long-term health risks

364 in ART offspring. Our study highlights ART clinical interventions that require particular

365 surveillance. More effort should be expended to optimize current ART systems, and the choice of

366 appropriate procedures requires careful evaluation. Since epigenomic changes might be

367 maintained throughout the human $\operatorname{lifespan}^{44}$ and can potentially be transmitted to subsequent

368 generations, long-term follow-up and health evaluation of ART offspring are necessary to provide

369 more robust clinical evidence. 
Methods

373 Methods and any associated references are available in the supplemental sections.

\section{Acknowledgments}

376 We thank the families participated in this study. We thank Robert Norman for discussion and

377 reviewing the manuscript. This project is funded by National Natural Science Foundation of China

378 (81730038; 81521002), National Key Research and Development Program (2018YFC1004000; 379 2017YFA0103801; 2017YFA0105001) and Strategic Priority Research Program of the Chinese 380 Academy of Sciences (XDA16020703). Y.W. was supported by Postdoctoral Fellowship of 381 Peking-Tsinghua Center for Life Science.

\section{Author contributions}

W.C., X.M., Y.P. and S.K. wrote the manuscript. W.C. and X.M. collected the study materials and

387 and performed bioinformatic analysis. J.Q., L.Y. and Y.Wang. developed the experimental

\section{Competing interests}

391 The authors declare no competing interests. 


\section{References}

1. Fauser, B.C. Towards the global coverage of a unified registry of IVF outcomes. Reproductive biomedicine online 38, 133-137 (2019).

2. Winston, R.M. \& Hardy, K. Are we ignoring potential dangers of in vitro fertilization and related treatments? Nat. Med. 8, 14-18 (2002).

3. Esteves, S.C., Roque, M., Bedoschi, G., Haahr, T. \& Humaidan, P. Intracytoplasmic sperm injection for male infertility and consequences for offspring. Nat. Rev. Urol. 15, 535-562 (2018).

4. Ludwig, M., Schröder, A.K. \& Diedrich, K. Impact of intracytoplasmic sperm injection on the activation and fertilization process of oocytes. Reprod. Biomed. Online 3, 230-240 (2001).

5. Kopeika, J., Thornhill, A. \& Khalaf, Y. The effect of cryopreservation on the genome of gametes and embryos: principles of cryobiology and critical appraisal of the evidence. Human reproduction update 21, 209-227 (2014).

6. Fleming, T.P., et al. Origins of lifetime health around the time of conception: causes and consequences. Lancet 391, 1842-1852 (2018).

7. Fruchter, E., et al. Health and functioning of adolescents conceived by assisted reproductive technology. Fertility and sterility 107, 774-780 (2017).

8. Kuiper, D., et al. Cardiovascular health of 9-year-old IVF offspring: no association with ovarian hyperstimulation and the in vitro procedure. Human Reproduction 32, 2540-2548 (2017).

9. Watanabe, N., et al. Is in vitro fertilization associated with preeclampsia? A propensity score matched study. BMC pregnancy and childbirth 14, 69 (2014).

10. Berntsen, S., et al. The health of children conceived by ART:'the chicken or the egg?'. Hum. Reprod. Update 25, 137-158 (2019).

11. Scherrer, U., Rexhaj, E., Allemann, Y., Sartori, C. \& Rimoldi, S.F. Cardiovascular dysfunction in children conceived by assisted reproductive technologies. European heart journal 36, 1583-1589 (2015).

12. Feil, R. \& Fraga, M.F. Epigenetics and the environment: emerging patterns and implications. Nat. Rev. Genet. 13, 97-109 (2012).

13. Zhu, P., et al. Single-cell DNA methylome sequencing of human preimplantation embryos. Nat. Genet. 50, 12-19 (2018).

14. $\mathrm{Xu}, \mathrm{Q}$. \& Xie, W. Epigenome in early mammalian development: inheritance, reprogramming and establishment. Trends Cell Biol. 28, 237-253 (2018).

15. Gluckman, P.D., Hanson, M.A., Cooper, C. \& Thornburg, K.L. Effect of in utero and early-life conditions on adult health and disease. N. Engl. J. Med. 359, 61-73 (2008).

16. Lazaraviciute, G., Kauser, M., Bhattacharya, S., Haggarty, P. \& Bhattacharya, S. A systematic review and metaanalysis of DNA methylation levels and imprinting disorders in children conceived by IVF/ICSI compared with children conceived spontaneously. Hum. Reprod. Update 20, 840-852 (2014).

17. Song, S., et al. DNA methylation differences between in vitro-and in vivo-conceived children are associated with ART procedures rather than infertility. Clin. Epigenetics 7, 41 (2015).

18. Ghosh, J., Coutifaris, C., Sapienza, C. \& Mainigi, M. Global DNA methylation levels are altered by modifiable clinical manipulations in assisted reproductive technologies. Clin. Epigenetics 9, 14 (2017).

19. Melamed, N., Choufani, S., Wilkins-Haug, L.E., Koren, G. \& Weksberg, R. Comparison of genome-wide and 
gene-specific DNA methylation between ART and naturally conceived pregnancies. Epigenetics 10, 474-483 (2015).

20. Estill, M.S., et al. Assisted reproductive technology alters deoxyribonucleic acid methylation profiles in bloodspots of newborn infants. Fertility and sterility 106, 629-639. e610 (2016).

21. El Hajj, N., et al. DNA methylation signatures in cord blood of ICSI children. Human Reproduction 32, 17611769 (2017).

22. Choufani, S., et al. Impact of assisted reproduction, infertility, sex and paternal factors on the placental DNA methylome. Human Molecular Genetics 28, 372-385 (2018).

23. Castillo-Fernandez, J.E., et al. DNA methylation changes at infertility genes in newborn twins conceived by in vitro fertilisation. Genome Med. 9, 28 (2017).

24. Tobi, E.W., et al. DNA methylation signatures link prenatal famine exposure to growth and metabolism. Nat. Commun. 5, 5592 (2014).

25. Bauer, T., et al. Environment - induced epigenetic reprogramming in genomic regulatory elements in smoking mothers and their children. Mol. Syst. Biol. 12, 861 (2016).

26. Kaminsky, Z.A., et al. DNA methylation profiles in monozygotic and dizygotic twins. Nat. Genet. 41, 240-245 (2009).

27. Gordon, L., et al. Neonatal DNA methylation profile in human twins is specified by a complex interplay between intrauterine environmental and genetic factors, subject to tissue-specific influence. Genome Res. 22, 1395-1406 (2012).

28. Tournaye, H. Male factor infertility and ART. Asian J. Androl. 14, 103-108 (2012).

29. Sahai, E. \& Marshall, C.J. RHO-GTPases and cancer. Nat. Rev. Cancer 2, 133-142 (2002).

30. Cohen, M.E. The brave new baby and the law: fashioning remedies for the victims of in vitro fertilization. Am. J. Law Med. 4, 319-336 (1978).

31. Liu, Y., et al. Comparison of Genome-Wide DNA Methylation Profiles of Fetal Tissues Conceived by In Vitro Fertilization and Natural Conception (October 16, 2018). Available at SSRN: https://ssrn.com/abstract=3267672

32. Pelkonen, S., et al. Physical health of singleton children born after frozen embryo transfer using slow freezing: a 3-year follow-up study. Human Reproduction 30, 2411-2418 (2015).

33. Kuiper, D.B., et al. Asthma and asthma medication use among 4-year-old offspring of subfertile couplesassociation with IVF? Reproductive biomedicine online 31, 711-714 (2015).

34. Wei, D., et al. Frozen versus fresh single blastocyst transfer in ovulatory women: a multicentre, randomised controlled trial. The Lancet 393, 1310-1318 (2019).

35. Saso, J., Shields, S.-K., Zuo, Y. \& Chakraborty, C. Role of Rho GTPases in human trophoblast migration induced by IGFBP1. Biol. Reprod. 86, 1-9 (2012).

36. Redman, C.W. \& Sargent, I.L. Latest advances in understanding preeclampsia. Science 308, 1592-1594 (2005).

37. Guo, X.-Y., et al. Cardiovascular and metabolic profiles of offspring conceived by assisted reproductive technologies: a systematic review and meta-analysis. Fertility and sterility 107, 622-631. e625 (2017).

38. Alberti, K.G.M., Zimmet, P. \& Shaw, J. The metabolic syndrome-a new worldwide definition. The Lancet 366, 1059-1062 (2005).

39. Chen, X.-K., et al. In vitro fertilization is associated with an increased risk for preeclampsia. Hypertension in pregnancy 28, 1-12 (2009). 
bioRxiv preprint doi: https://doi.org/10.1101/816157; this version posted October 24,2019 . The copyright holder for this preprint (which was not certified by peer review) is the author/funder, who has granted bioRxiv a license to display the preprint in perpetuity. It is made available under aCC-BY-NC 4.0 International license.

40. Williams, C.L., et al. Cancer risk among children born after assisted conception. New England Journal of Medicine 369, 1819-1827 (2013).

41. Sandin, S., Nygren, K.-G., Iliadou, A., Hultman, C.M. \& Reichenberg, A. Autism and mental retardation among offspring born after in vitro fertilization. Jama 310, 75-84 (2013).

42. Canovas, S., et al. DNA methylation and gene expression changes derived from assisted reproductive technologies can be decreased by reproductive fluids. Elife 6, e23670 (2017).

43. Tan, K., et al. Impaired imprinted $X$ chromosome inactivation is responsible for the skewed sex ratio following in vitro fertilization. Proc. Natl. Acad. Sci. U. S. A. 113, 3197-3202 (2016).

44. Dominguez-Salas, P., et al. Maternal nutrition at conception modulates DNA methylation of human metastable epialleles. Nature communications 5, 3746 (2014).

\section{Major Figures and Figure Legends:}
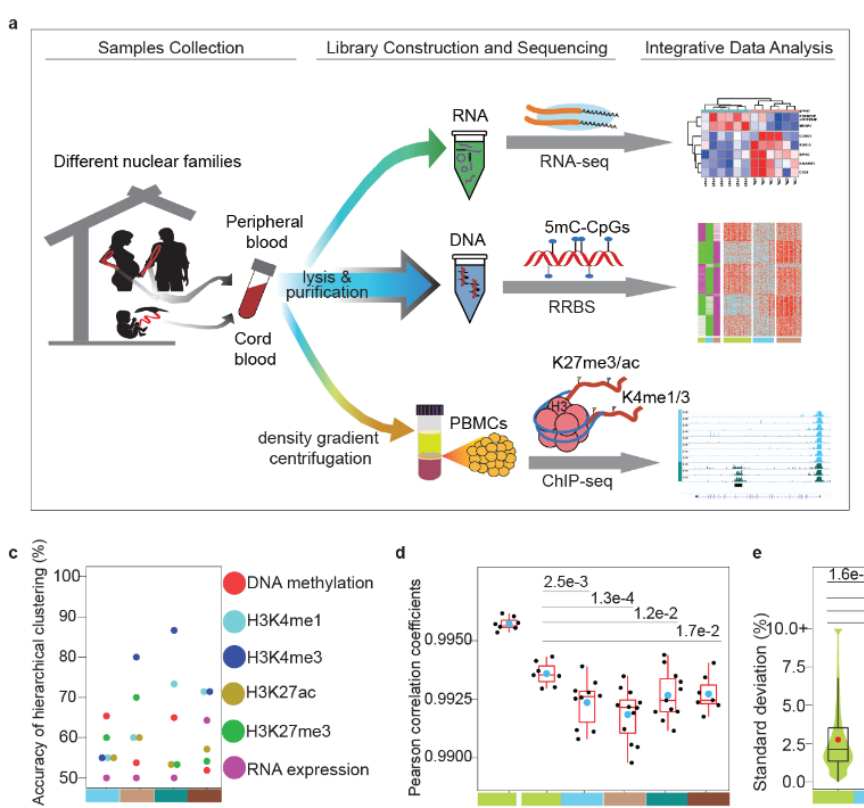

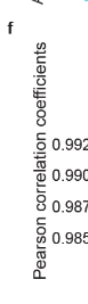

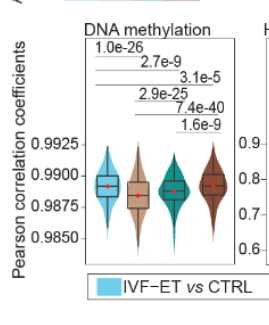

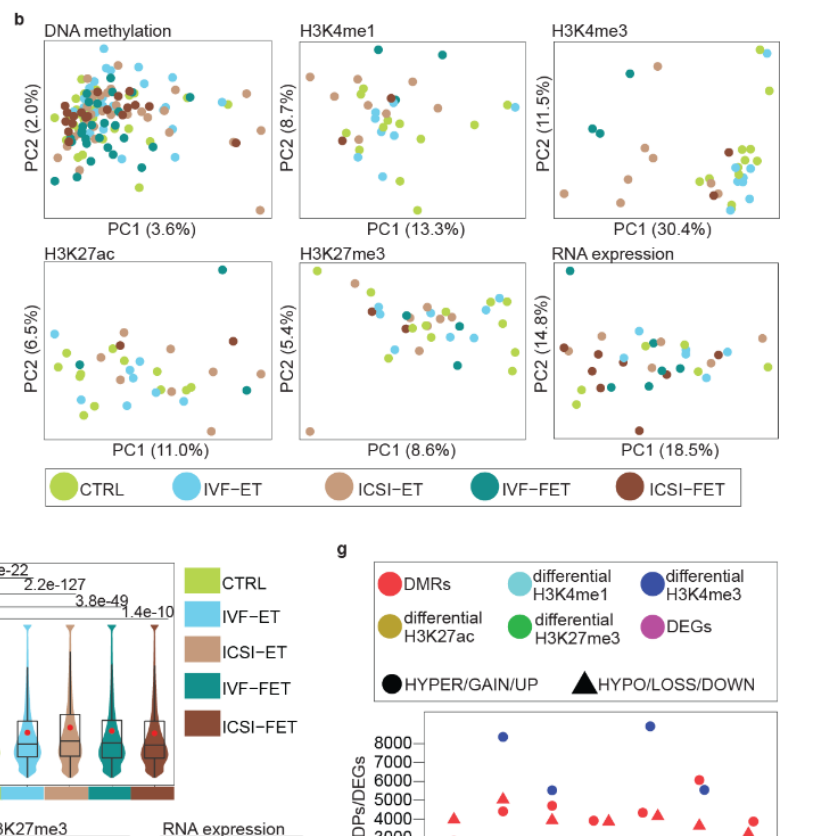
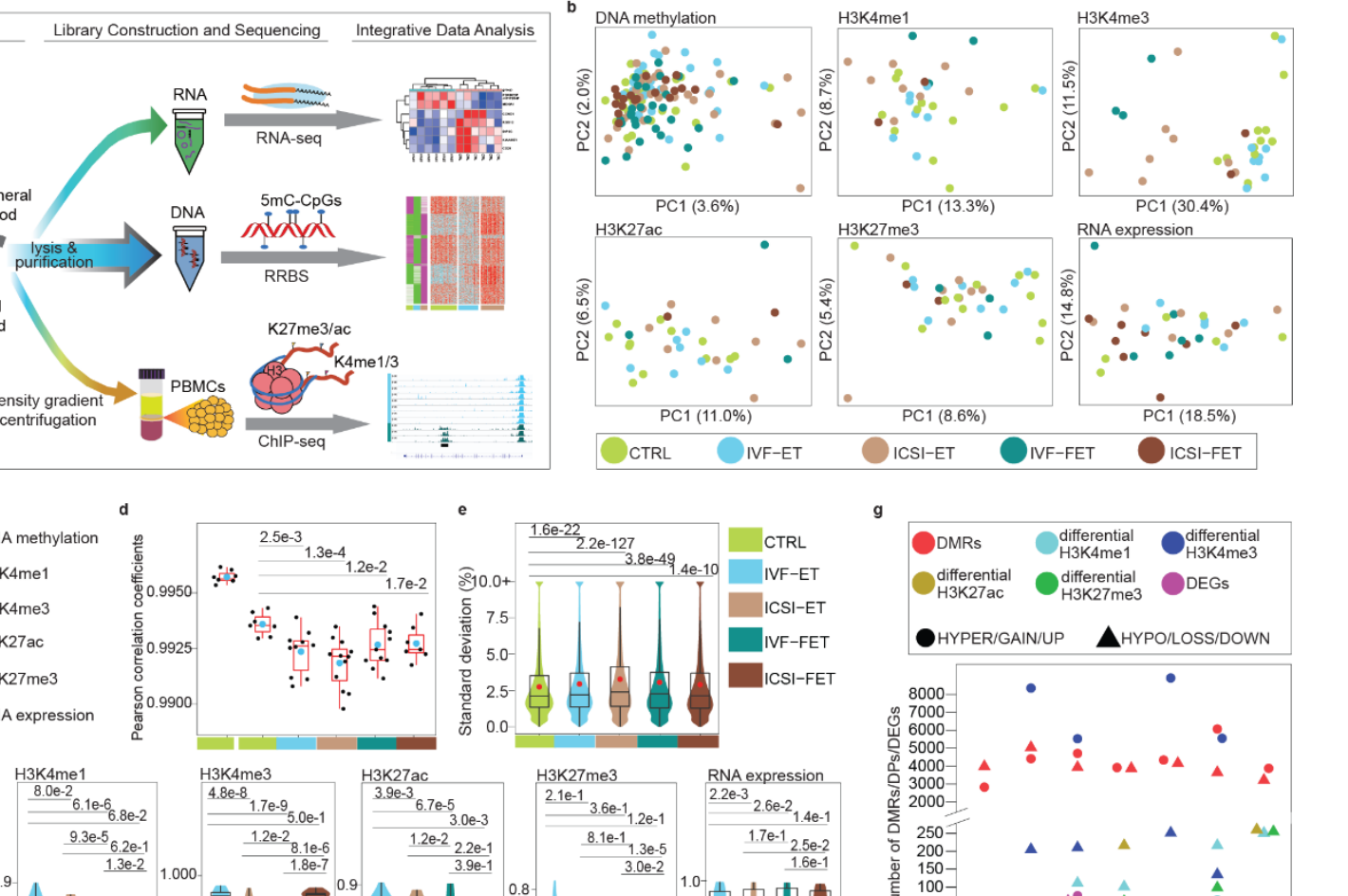

4.8e-8 3

$\frac{1.7 \mathrm{e}-9}{5.0 \mathrm{e}-1}$

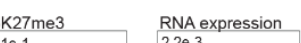

2.1e-1 3

ICSI-ET vS CTRL

IVF-FET vs CTR

ICSI-FET vs CTRL

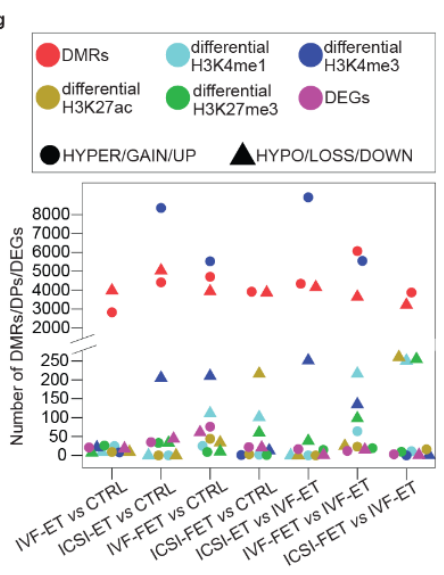

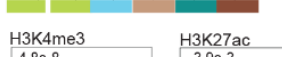
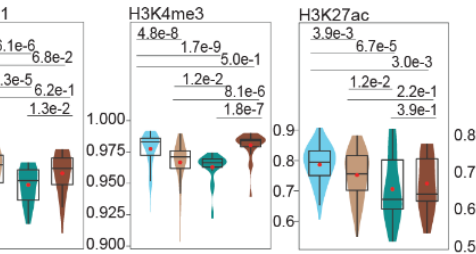
Fig. 1, Epigenome profiling in ART-conceived neonates. a. Graphical overview of the study design. b. Principal component analysis (PCA) for all the five groups (CTRL, IVF-ET, ICSI-ET, IVF-FET, and ICSI-FET) of neonatal samples by using each layer of the reference epigenome and transcriptome. For DNA methylation, only the 100-bp tiles covered all neonatal samples were used; ChIP-seq peaks of all neonatal samples for each histone modification were pooled together, then the overlapping peaks were merged; genes with $\mathrm{TPM}=0$ in all neonatal samples are removed. Finally, the number of genomic regions for each layer to generate the PCAs: DNA methylation (467097 100-bp tiles), H3K4me1 (195570 peaks), H3K4me3 (86157 peaks), H3K27ac (324900 peaks), H3K27me3 (257175 peaks), and RNA expression (26326 genes). c. The accuracy of hierarchical clustering for IVF-ET vs CTRL (cyan), ICSI-ET vs CTRL (sienna), IVF-FET vs CTRL (dark cyan), and ICSI-FET vs CTRL (dark sienna) neonatal samples. Samples and genomic regions used were the same as in b. d. Box plots for the distribution of the within-twin-pair Pearson correlation coefficients for genomewide DNA methylation in each group as in $\mathbf{b}$, respectively. Twin pairs in CTRL group were classified into two subgroups, monozygotic and dizygotic twins. Each black dot represents the Pearson correlation coefficient and the cyan dots are the arithmetic means. The p-value between two groups was determined by unpaired and two-tailed t-test. e. Violin-box plots showed the distribution of standard deviations of genomewide DNA methylation for neonatal samples in each group mentioned in $\mathbf{b}$, respectively. The p-value between two groups was determined by Wilcoxon rank-sum test. f. For each layer of the reference epigenome and transcriptome, violin-box plots showing the distribution of Pearson correlation coefficients between CTRL and one of the four ART groups. The red dots are the arithmetic means. The p-

512 value between two groups was determined by Wilcoxon rank-sum test. g. The number of DMRs, 513 DPs of four histone modifications, and DEGs for neonatal samples were shown for seven 514 comparisons: IVF-ET versus CTRL, ICSI-ET versus CTRL, IVF-FET versus CTRL, ICSI-FET 515 versus CTRL, ICSI-ET versus IVF-ET, IVF-FET versus IVF-ET, and ICSI-FET versus IVF-ET. 

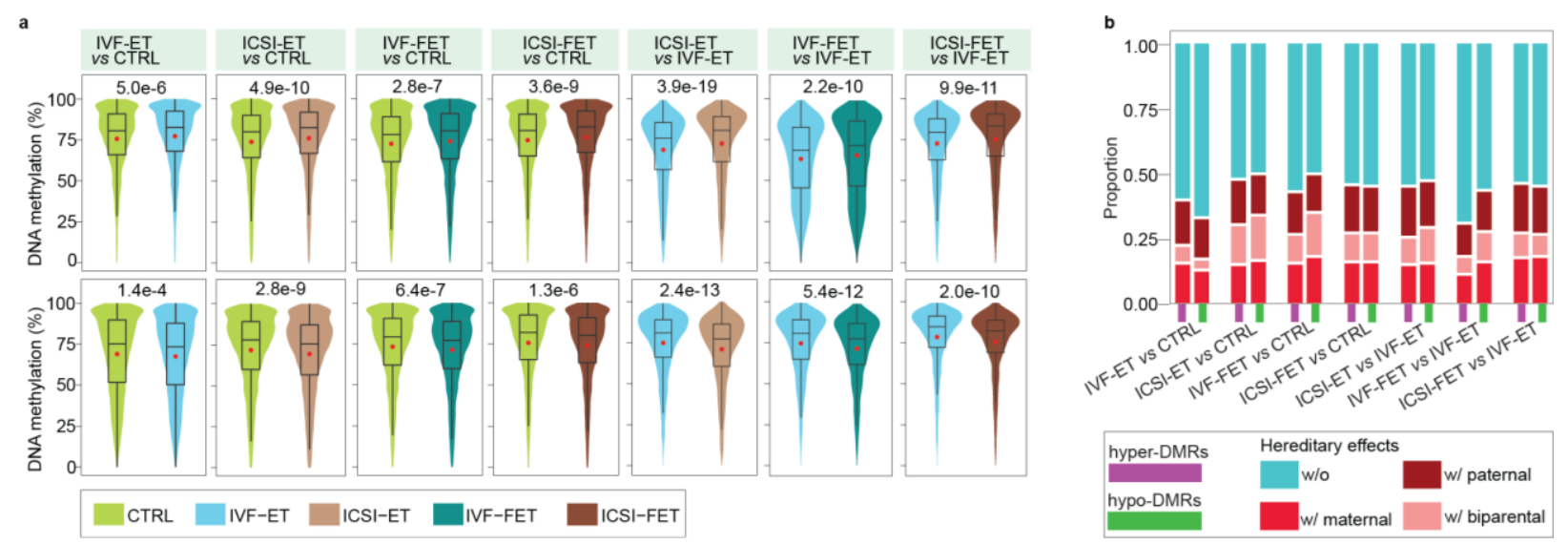

Fig. 2, Remove the parental effects in neonatal DMRs. a. Violin-box plots displayed the distribution of DNA methylation level in paternal samples at hyper- (upper) and hypo-DMRs (lower) of the seven comparisons, IVF-ET versus CTRL, ICSI-ET versus CTRL, IVF-FET versus CTRL, ICSI-FET versus CTRL, ICSI-ET versus IVF-ET, IVF-FET versus IVF-ET, and ICSI-FET versus IVF-ET neonatal samples. The p-value between two groups was determined by Wilcoxon rank-sum test. b. Bar graph showed the proportion of DMRs with/without hereditary effects in the hyper- or hypo-DMRs of each comparison. Any one of the 14 groups of DMRs were classified into four subgroups, without hereditary effects (w/o), only with paternal hereditary effects

527 (w/paternal, only overlapped with the DMRs of comparison for the corresponding fathers), only with maternal hereditary effects (w/ maternal, only overlapped with the DMRs of comparison for the corresponding mothers), and with biparental hereditary effects (w/ biparental, overlapped with the DMRs of comparisons for the corresponding fathers and mothers). 


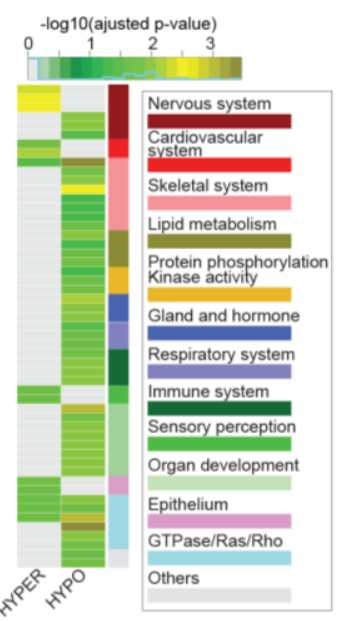

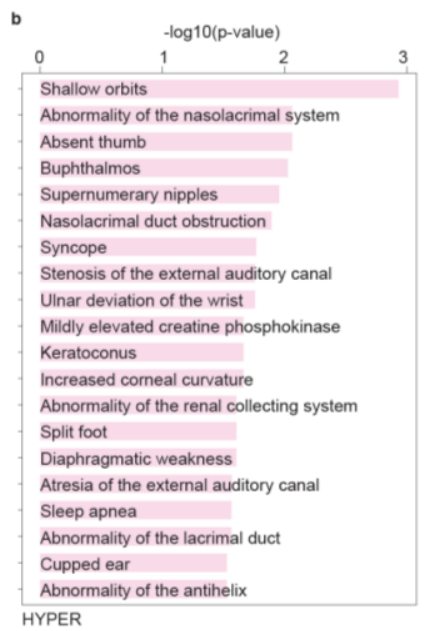

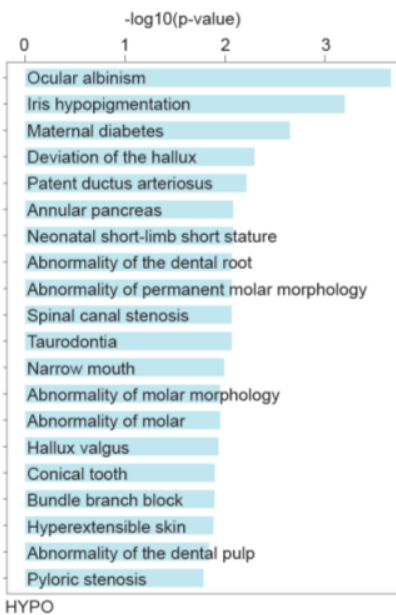

HYPO

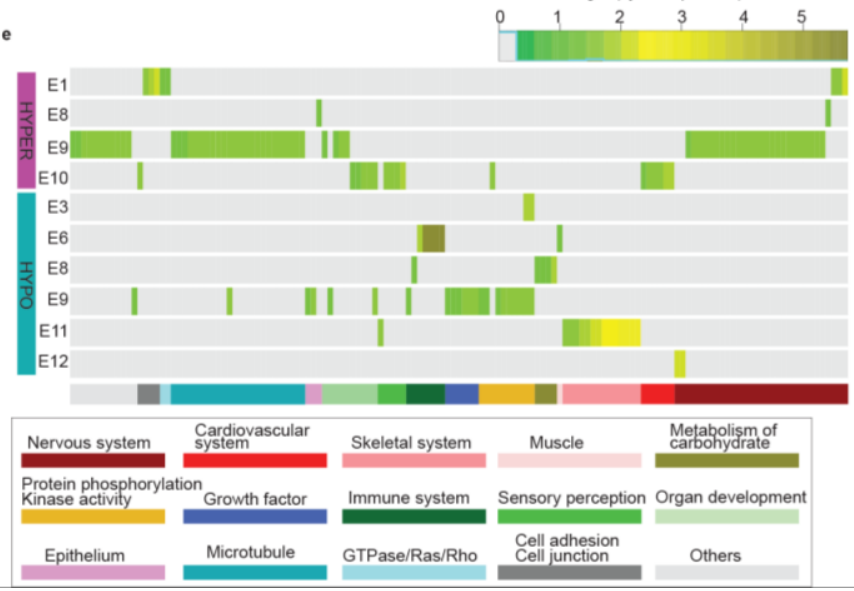

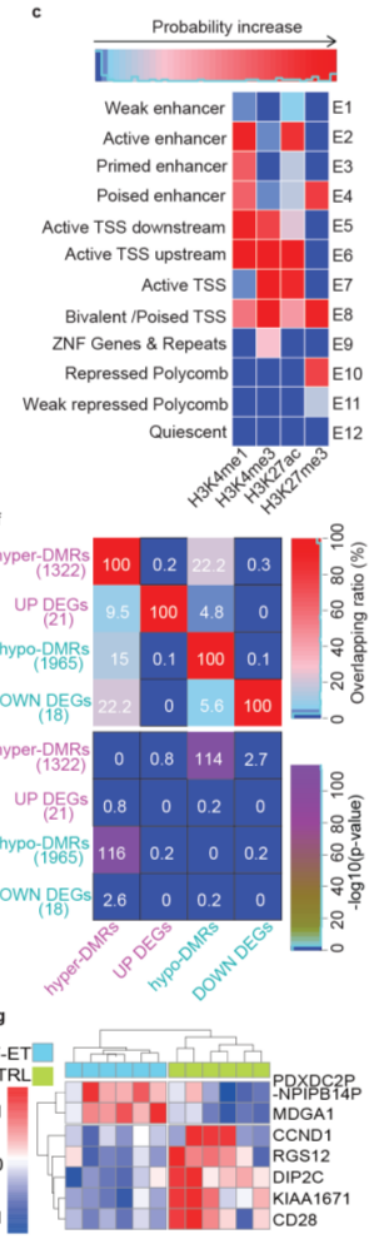

Fig. 3, IVF effects on the epigenome of the offspring. a. Gene ontology (biological process) analysis for the hyper- and hypo-DMRs of IVF-ET versus CTRL, all enrichment terms with adjusted $p$-value $<0.05$ were grouped and shown. b. The top 20 enriched ontology terms of human phenotype for hyper- (left) and hypo-DMRs (right). c. Based on the four histone modifications in all neonatal samples, 12 chromatin states were defined by chromHMM. Each row of the heatmap corresponds to a specific chromatin state, and each column corresponds to a different histone mark, H3K4me1, H3K4me3, H3K27me3, or H3K27ac. d. In each neonatal sample of CTRL and IVF-

541 ET groups, column scaled fold enrichment of the chromatin states at genomewide regions 542 (Genome), RRBS covered regions (RRBS), hyper- and hypo-DMRs (HYPER and HYPO) was 543 shown. e. For each subgroup of DMRs in d, its gene ontology (biological process) enrichment 544 analysis results were grouped and shown, if its enrichment terms existed (adjusted p-value $<0.05$ ). 
545 f. Heatmap showed overlapping ratio among associated genes of DMRs and DEGs for IVF-ET vs 546 CTRL (upper), and their statistical significances (lower) determined by hypergeometric test. g.

547 Heatmap showed the expression level of DEGs overlapped with the associated genes of DMRs

548 (PDXDC2P-NPIPB14P, MDGA1, CCND1, RGS12, DIP2C, KIAA1671, CD28) among neonatal 549 samples in CTRL and IVF-ET. 
a

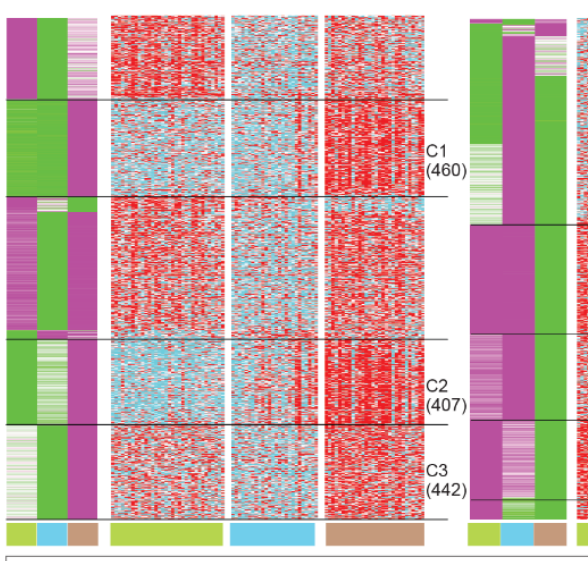

Row scaled DNA methylation level increase

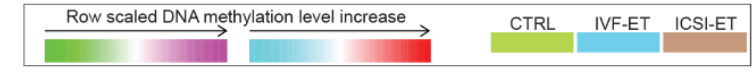

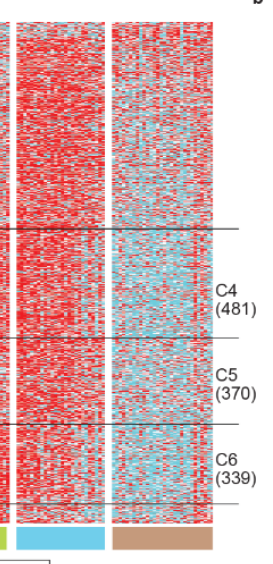
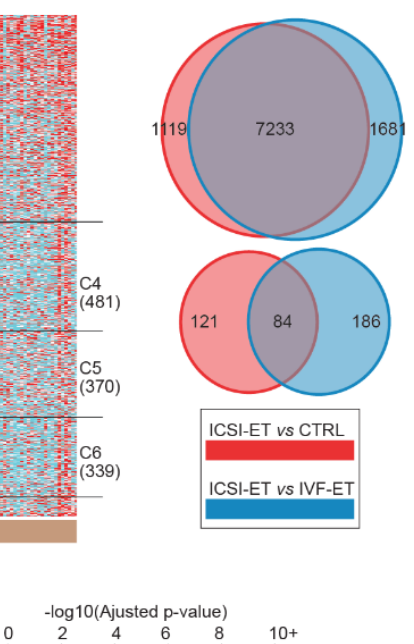

$10+$
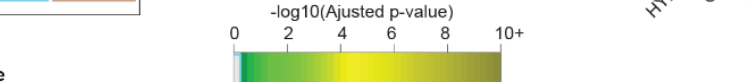

d

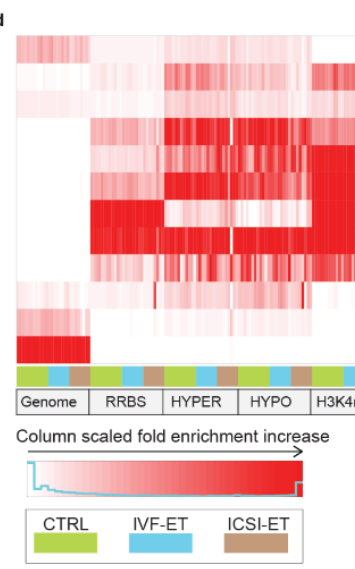

Fig. 4, Separate epigenetic effects of ICSI. a. K-means clustering for the hyper- (left) and hypoDMRs (right) between ICSI-ET vs IVF-ET neonatal samples. In green-purple heatmaps, the hyperDMRs (hypo-DMRs) were classified into seven (nine) clusters by k-means clustering on row scaled average DNA methylation level. With the same row order, row scaled DNA methylation level in each neonatal sample was also shown in cyan-red heatmap. The number of DMRs in the selected clusters were also shown. b. Venn diagram for the overlap between of the gain (upper) and lost (lower) H3K4me3 DPs of ICSI-ET vs CTRL, and ICSI-ET vs IVF-ET neonatal samples. (7233) and loss (84) H3K4me3 DPs, their gene ontology (biological process) enrichment analysis results were grouped and shown, if its enrichment terms existed (adjusted p-value $<0.05$ ). d. The 
563 chromatin states distribution of the selected hyper- and hypo-DMRs and H3K4me3 DPs (7233

564 GAIN, 84 LOSS) in each neonatal sample of CTRL, IVF-ET, and ICSI-ET. The chromatin states

565 were defined in fig. 3c. e. For DMRs or DPs in different states in d, their gene ontology (biological

566 process) enrichment analysis results were grouped and shown, if the enrichment terms existed

567 (adjusted p-value $<0.05)$.

568 
bioRxiv preprint doi: https://doi.org/10.1101/816157; this version posted October 24,2019 . The copyright holder for this preprint (which was not certified by peer review) is the author/funder, who has granted bioRxiv a license to display the preprint in perpetuity. It is made available under aCC-BY-NC 4.0 International license.

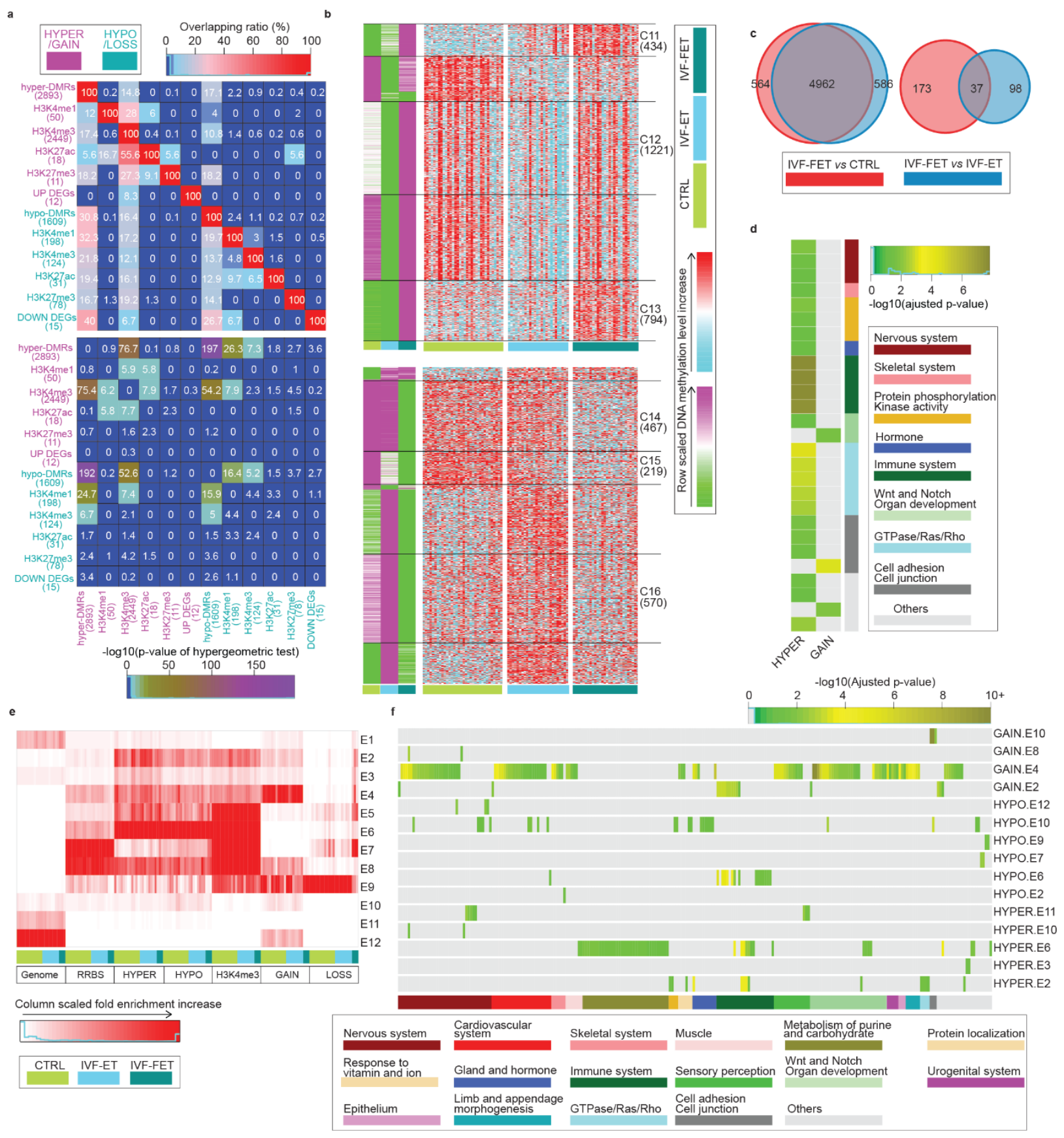

571 Fig. 5, Separate epigenetic effects of freezing-thaw. a. Overlapping ratio among DEGs,

572 associated genes of DMRs and DPs for IVF-FET vs IVF-ET neonatal samples (upper), and their

573 statistical significances (lower) determined by hypergeometric test. b. K-means clustering for the

574 hyper- (upper) and hypo-DMRs (lower) between IVF-FET vs IVF-ET neonatal samples. In green- 
575 purple heatmaps, the hyper-DMRs (hypo-DMRs) were classified into six (seven) clusters by k576 means clustering on row scaled average DNA methylation level. With the same row order, row 577 scaled DNA methylation level in each neonatal sample was also shown in cyan-red heatmap. The 578 number of DMRs in the selected clusters were also shown. c. Venn diagrams displayed the overlap 579 between the gain (left) and lost (right) H3K4me3 DPs of IVF-FET vs CTRL and that of IVF-FET 580 vs IVF-ET. d. For the selected hyper- (union of cluster C11-C13) and hypo-DMRs (union of cluster 581 C14-C16), overlapping gain (4962) and loss (37) H3K4me3 DPs, their gene ontology (biological 582 process) enrichment analysis results were grouped and shown, if the enrichment terms existed 583 (adjusted p-value $<0.05$ ). e. The chromatin states distribution of the selected hyper- and hypo584 DMRs, overlapping 4962 gain and 37 loss H3K4me3 DPs in each neonatal sample of CTRL, IVF585 ET, and IVF-FET. The chromatin states were defined in fig. 3c. f. For DMRs or DPs in different 586 states in e, all enrichment terms of gene ontology (biological process) with adjusted p-value $<0.05$ 587 were grouped and shown. 
bioRxiv preprint doi: https://doi.org/10.1101/816157; this version posted October 24,2019 . The copyright holder for this preprint (which was not certified by peer review) is the author/funder, who has granted bioRxiv a license to display the preprint in perpetuity. It is made available under aCC-BY-NC 4.0 International license.

a
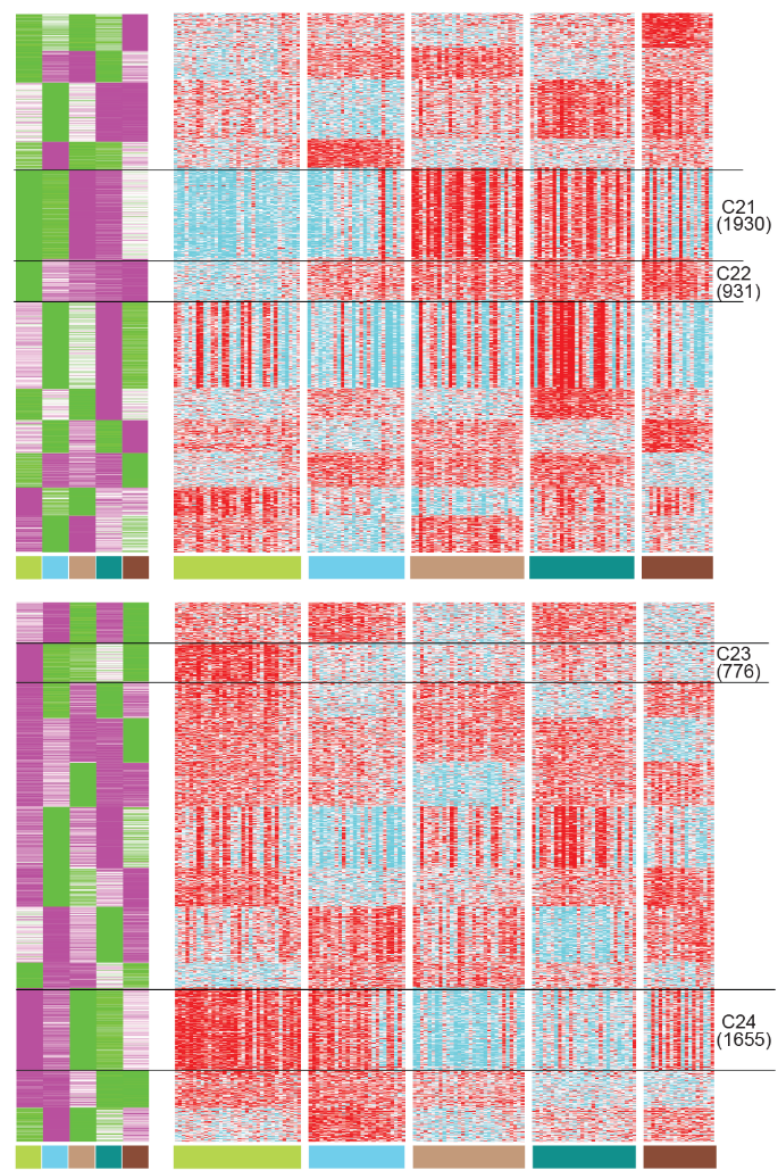

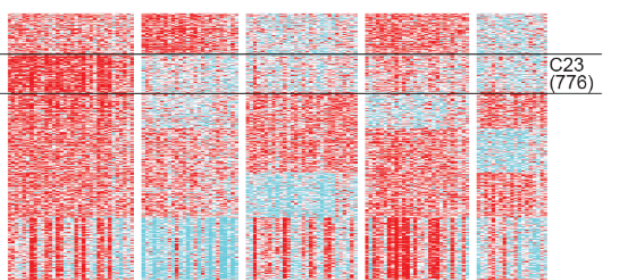

Row scaled DNA methylation level increase

$\mathrm{C} 21 \mathrm{C} 22 \mathrm{C} 23 \mathrm{C} 24$

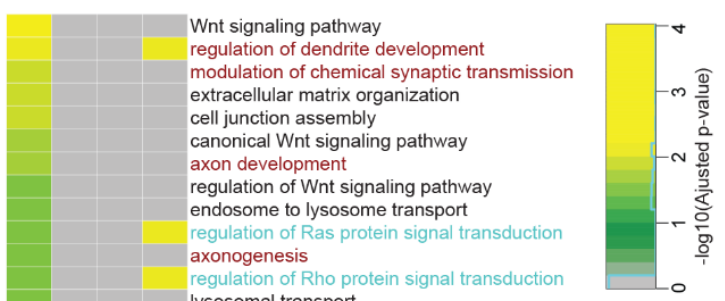

regulation of AMPA receptor activity

vacuolar transport

regulation of GTPase activity

positive regulation of stress-activated MAPK cascade positive regulation of stress-activated kinase signaling response to peptide

regulation of small GTPase mediated signal transduction regulation of cell morphogenesis

regulation of neuron projection development

positive regulation of neurogenesis

positive regulation of cell projection organization

respiratory system development

cell-cell adhesion via plasma-membrane adhesion molecules positive regulation of neuron projection development positive regulation of neuron differentiation

respiratory tube development

regulation of cell morphogenesis involved in differentiation positive regulation aortic valve morphogenesis

pulmonary valve morphogenesis Rho protein signal transduction
ventricular septum development
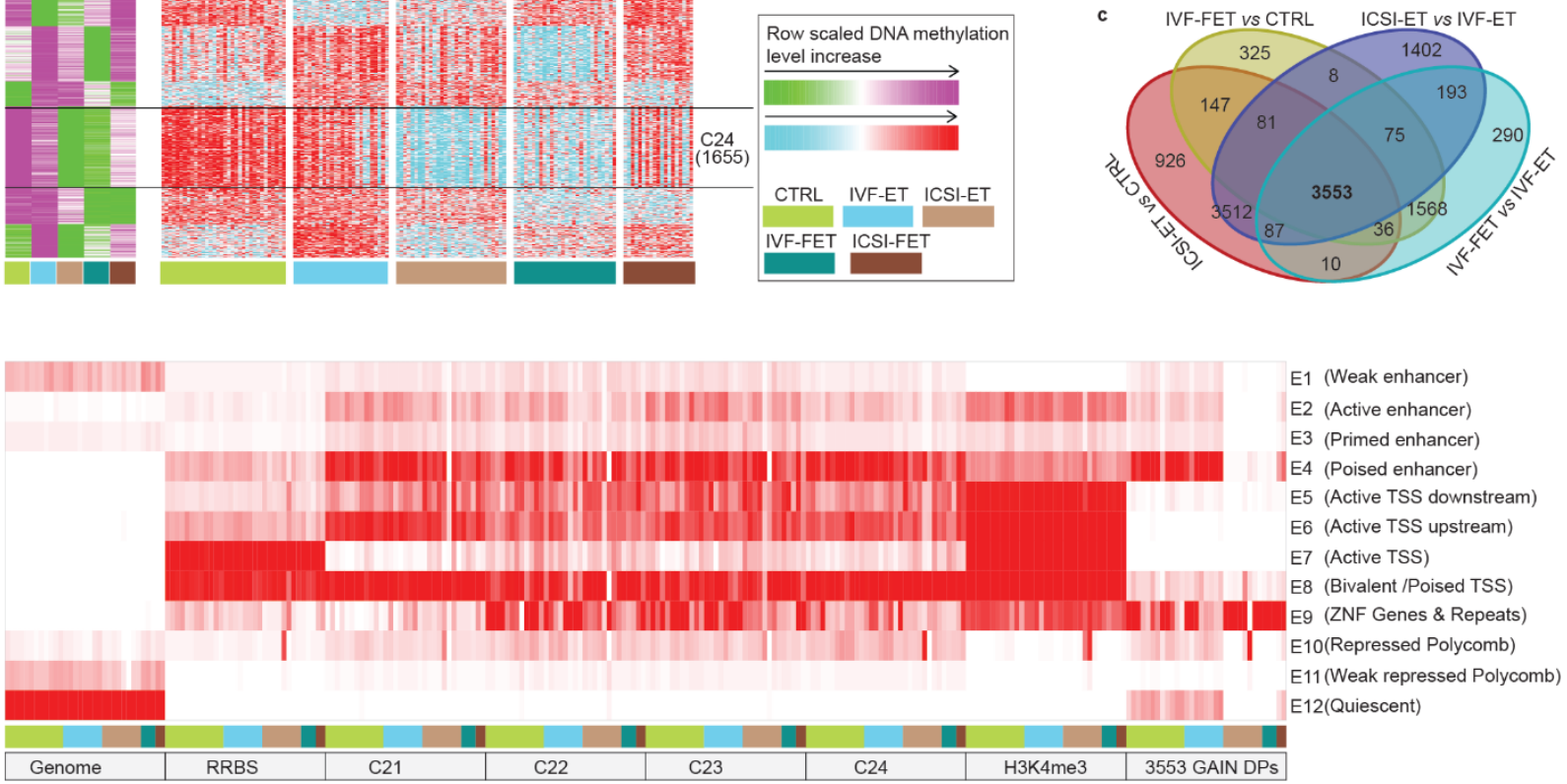

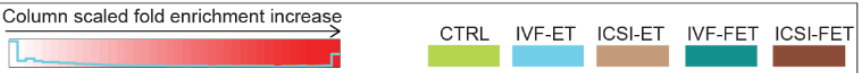

Fig. 6 Common Epigenetic effect of different ARTs.

a. K-means clustering for the all merged hyper- (up) and hypo-DMRs (down) of the seven comparisons. The hyper- and hypo-DMRs of the seven comparisons were merged if they have 1-bp common region at least, respectively. In greenpurple heatmaps, DMRs were classified into twelve clusters by k-means clustering on row scaled 
595 average DNA methylation level. With the same row order, row scaled DNA methylation level in 596 each neonatal sample was also shown in cyan-red heatmap. The number of DMRs in the selected 597 clusters were also shown. b. For the selected clusters of hyper-DMRs (C21, C22) and hypo-DMRs 598 (C23, C24), all enrichment terms of gene ontology (biological process) with adjusted p-value $<$ 5990.05 were grouped and shown. c. Venn diagrams displayed the overlap of the gain H3K4me3 DPs 600 for ICSI-ET vs CTRL, IVF-FET vs CTRL, ICSI-ET vs CTRL, and IVF-FET vs IVF-ET. d. The 601 chromatin states distribution in each neonatal sample for the selected DMRs and overlapping gain 602 H3K4me3 DPs. The chromatin states were defined in fig. 3c. 